\title{
Soluble Receptor for Advanced Glycation End Products (sRAGE) is Up-Regulated in Multiple Sclerosis Patients Treated with Interferon $\boldsymbol{\beta}$-1a
}

\author{
Mahnoosh Rahimia Sarah Saadat Aghabozorg Afjeha Mir Davood Omrania,b \\ Shahram Arsang-Jang ${ }^{c}$ Maziar Ganji ${ }^{a}$ Rezvan Noroozid ${ }^{d}$ Mohammad Taheria,b \\ Soudeh Ghafouri-Farda,b \\ aDepartment of Medical Genetics, Shahid Beheshti University of Medical Sciences, Tehran, bUrogenital \\ Stem Cell Research Center, Shahid Beheshti University of Medical Sciences, Tehran, 'Department of \\ Biostatistics and Epidemiology, Qom University of Medical Sciences, Tehran, dPhytochemistry research \\ center, Shahid Beheshti University of Medical Sciences, Tehran, Iran
}

\section{Key Words}

sRAGE • Multiple sclerosis

\begin{abstract}
Background/Aims: Multiple sclerosis (MS) is a chronic inflammatory disorder of the central nervous system. Considering the role of immune system in its pathogenesis, researchers have focused on evaluation of the expression of immune-related genes or proteins in MS patients. Among proteins whose participation in inflammatory process has been documented is the receptor for advanced glycation end products (RAGE). Methods: In the present study, we compared RAGE transcript levels by means of quantitative real-time PCR as well as the serum level of soluble RAGE (sRAGE) by means of enzyme- linked immunosorbent assay (ELISA) in 50 IFN $\beta$-1a responsive relapsing-remitting MS patients when compared with age and sexmatched healthy subjects. Results: Elevated expression of RAGE as well as higher levels of sRAGE were detected in IFN- $\beta$ responsive MS patients compared with the controls. A significant inverse correlation between SRAGE plasma concentrations and the expanded disability status scale (EDSS) was also detected in which each unit of increase in SRAGE level resulted in a 0.308 unit decrease in EDSS. Conclusion: Considering the stable clinical state of the MS patients in this study and their response to IFN $\beta-1 a$, the elevated levels of sRAGE in patients compared with healthy subjects could be related to the effects of this kind of treatment.
\end{abstract}

M. Rahimi and S. S. Aghabozorg Afjeh contributed equally to this work.

Mohammad Taheri

and Soudeh Ghafouri-Fard

KARGER
Department of Medical Genetics, Shahid Behesht

University of Medical Sciences, Tehran 19857-17443 (Iran)

Tel.00982123872572,E-Mail mohammad_823@yahoo.com, s.ghafourifard@sbmu.ac.ir 


\section{Introduction}

Multiple sclerosis (MS) as a chronic inflammatory disorder of the central nervous system (CNS) [1] is believed to be a consequence of the interaction of numerous genetic and environmental factors [2-4]. Considering the role of immune system in the development of MS, researchers have focused on evaluation of the expression of immune-related genes or proteins in MS patients. Among proteins for which participation in the inflammatory process has been documented is the receptor for advanced glycation end products (RAGE). This transmembrane protein is a receptor in the immunoglobulin superfamily which binds to several ligands. RAGE-ligand interaction results in up-regulation of cytokines, chemokines, and adhesion molecules with possible roles in the commencement and continuation of inflammation [5].

RAGE expression has been documented in a range of cells and tissues, including in endothelial cells, smooth muscle cells, mononuclear phagocytes and neurons [6]. Ligand/ RAGE interaction and subsequent RAGE over-expression trigger downstream signaling pathways resulting in increased release of reactive oxygen species and boosts production of proinflammatory cytokines [7]. Over-expression of RAGE has been detected in several immune-related disorders, including vascular [8] and lung inflammation [9] and rapid neurodegeneration in infantile neuronal ceroid lipofuscinosis [10]. Increased levels of RAGE have been detected in the neurons and inflammatory cells of spinal cord tissues originating from MS patients compared to those of the healthy controls [11]. In addition, suppression of RAGE expression decreases penetration of immune and inflammatory cells into the CNS and has been seen to reverse the inflammatory process in experimental autoimmune encephalomyelitis (EAE) [11].

Considering the putative role of RAGE in the pathogenesis of MS, in the present casecontrol study we compared relative expression of RAGE transcripts as well as soluble RAGE (sRAGE) protein in MS patients and healthy subjects. In addition to full-length membranebound RAGE, which has extracellular, transmembrane and cytosolic domains, two other forms of RAGE are produced either through alternative splicing of RAGE mRNA (N-truncated RAGE) or by proteolytic cleavage of membrane bound RAGE protein (C-truncated RAGE). The absence of transmembrane and cytosolic domains leads to extracellular release of these forms [12]. sRAGE exerts a decoy function through competition with membrane-bound RAGE for ligand binding which leads to reversal of the inflammatory effect of RAGE activation [8].

\section{Materials and Methods}

Patients and healthy subjects

Fifty unrelated patients with sporadic relapsing-remitting MS (RRMS) from Tehran Hospitals and the MS Society of Iran and 50 healthy matched controls participated in the current study. All MS patients had clinically definite (CD) RRMS based on the revised McDonald criteria [13]. At the time of sampling, all patients were in remission and clinically stable.

The exclusion criteria were the detection of an insufficient vitamin D level and the presence of HLADRB1*15 in patients. In addition, patients or healthy subjects with any inflammatory or autoimmune disease (other than MS), a history of diabetes mellitus, cancer, acute or chronic infection, cardiovascular disease or any other systematic disorder, type 1-4 hypersensitivities, current smoking, or history of treatment with immunosuppressive and immunomudulatory drugs (except for

Table 1. Nucleotide sequence of primers and probes used in the study

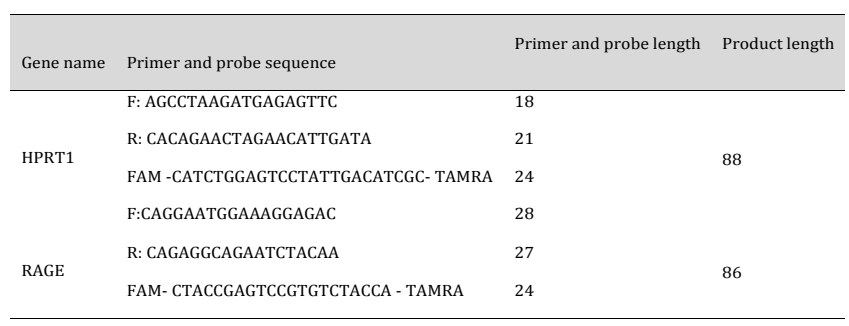


IFN $\beta$-1a for case group) were excluded from the study. Patients were responsive to IFN $\beta$-1a treatment (intramuscular injection of $20 \mu \mathrm{g}$ of CinnoVex (CinnaGen; Iran) three times a week) over the course of two years of follow-up. They showed either no progression in the Expanded Disability Status Scale (EDSS) or no relapse during the follow-up period.

\section{RNA extraction and real-time PCR}

Total RNA was extracted from the blood samples of all participants using a hybrid-RTM blood RNA extraction kit (GeneAll; Korea) according to manufacturer instructions. Subsequently, a high-capacity cDNA reverse transcription kit (Applied Biosystems; USA) was used to convert RNA to single-strand cDNA. The nucleotide sequences of probes and primers for RAGE and HPRT1 amplification which have been designed in Allele ID 7 software (Premier Biosoft; USA) are shown in Table 1. The latter gene was used to normalize the expression of RAGE in patients and controls. Real-time quantitative PCR was carried out in the Corbett Rotor gene 6000 (Qiagen; Australia) using TaqMan Universal PCR Master Mix (Applied Biosystems; USA). A negative control (with no cDNA template) was included in each run to determine any possible contamination. The PCR program included $5 \mathrm{~min}$ at $95^{\circ} \mathrm{C}$ and a subsequent 40 cycles at $95^{\circ} \mathrm{C}$ for $15 \mathrm{sec}$ and $60^{\circ} \mathrm{C}$ for $1 \mathrm{~min}$.

\section{Soluble RAGE (sRAGE) plasma concentration}

The RAGE plasma concentration was evaluated using the human soluble receptor for advanced glycation end products (sRAGE) enzyme-linked immunosorbent assay (ELISA) kit (Bioassay Technology; China) used to assay sRAGE on the basis of Biotin double antibody sandwich technology. The sRAGE values are shown in $\mathrm{pg} / \mathrm{ml}$ and defined as mean \pm SEM (standard error of mean).

\section{Statistical analysis}

SPSS 18 for Windows (SPSS; USA) was used for statistical analysis. To test the significance of the difference in means between groups, Kruschke's Bayesian estimation was used to fit a two-sample Bayesian t-test. A normal prior distribution was assumed for parameters with 200000 iterations. The Bayesian independent $t$-test was used as an alternative to the t-test to obtain more information about the samples and the difference in means compared with a simple p-value. The Spearman correlation coefficient was applied for evaluation of correlations between the level of RAGE expression and clinical or demographic variables. The level of significance was fixed at $<0.05$.

\section{Results}

General data of study participants

The study consisted of 50 RRMS

patients aged 17 to 55 years and 50 healthy subjects aged 22 to 60 years. General information about study participants are summarized in Table 2 .

Table 2. General information of study participants

\section{RAGE relative transcript levels in patients and controls}

Real-time PCR with primers specific for exons 3 and 4 of RAGE showed significant upregulation of $R A G E$ in MS patients compared to healthy subjects (Table 3 ). Fig. 1 shows the relative RAGE expression in patients and the control group.

Soluble RAGE (SRAGE) plasma concentration The mean plasma concentration of sRAGE as assessed by the ELISA was significantly higher in MS patients than in the controls and

\begin{tabular}{lll}
\hline Variables & MS patients & Controls \\
\hline Female/Male [no. (\%)] & $39(78 \%) / 11(22 \%)$ & $37(74 \%) / 13(26 \%)$ \\
Age (mean \pm SD, Y) & $36.2 \pm 2.9$ & $35.3 \pm 2.1$ \\
Age of onset (mean \pm SD, Y) & $31.41 \pm 2.8$ & - \\
Relapsing-remitting course (no. \%) & $100(100 \%)$ & - \\
Disease duration (mean \pm SD, Y) & $4.58 \pm 3.2$ & - \\
EDSS (mean \pm SD) & $3.07 \pm 2.7$ & - \\
\hline
\end{tabular}

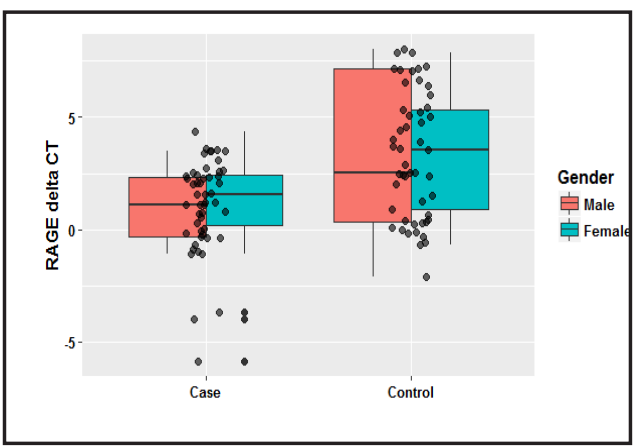

Fig. 1. Relative expression of RAGE in MS patients and healthy subjects. 
in both genders when compared with the sex-matched controls $(\mathrm{p}<0.001)$ (Table 4). Fig. 2 shows the sRAGE concentrations in patients and healthy subjects.

Correlation of RAGE transcript levels with patients' characteristics

The RAGE transcript levels correlated with age at disease onset $(\mathrm{r}=-0.358 ; \mathrm{p}=$ 0.011 ) and disease duration in patients $(\mathrm{r}$ $=0.281 ; \mathrm{p}=0.048)$, but not with EDSS $(\mathrm{r}=$ $-0.211 ; \mathrm{p}=0.141)$.

\section{Correlation of SRAGE plasma concentrations with patient} characteristics

Spearman correlation analysis showed a significant inverse correlation between sRAGE plasma concentration and patient age ( $\mathrm{r}=-0.304 ; \mathrm{p}=0.002)$ as well as sRAGE plasma concentration and EDSS ( $r=-0.282 ; p=0.047)$, but not for sRAGE plasma concentration and disease duration ( $\mathrm{r}=0.079 ; \mathrm{p}=0.584)$ or sRAGE plasma concentration and age at disease onset $(r=$ -0.059; $\mathrm{p}=0.683$ ).

The multiple linear regression model was used to assess the correlation between sRAGE plasma concentrations and patient characteristics. After adjusting for the effects of all independent variables, EDSS was the only variable which showed a significant inverse correlation with sRAGE plasma concentrations such that each unit of increase in SRAGE levels resulted in a 0.308 unit decrease in EDSS. Table 5 shows that each unit of increase in age resulted in a 0.056 unit increase in SRAGE, but these changes are not statistically significant $(\mathrm{p}=$ 0.71). sRAGE expression in female patients was lower than in male patients, but this difference also was not significant $(\mathrm{p}=$ 0.535).

Correlation between RAGE transcript levels and A reverse correlation was found between RAGE transcript levels and sRAGE plasma concentrations $(\mathrm{r}=-0.204 ; \mathrm{p}=$ 0.007; Fig. 3).

\section{Discussion}

In the present study, primers specific for amplification of the region coding the extracellular domain of $R A G E$ were used to demonstrate the higher expression of RAGE in MS patients
Table 3. Relative expression of RAGE in total MS patients and age-/sex-related subgroups compared with corresponding healthy subjects

\begin{tabular}{|c|c|c|c|c|c|c|}
\hline & & Controls & MS patients & Expression ratio & P value & 95\% Highest Density Interval \\
\hline & Total & 50 & 50 & 3.1479 & $<0.0001$ & {$[1.11,3.14]$} \\
\hline & Male & 13 & 11 & 2.9508 & 0.068 & {$[-0.353,4.69]$} \\
\hline & Female & 37 & 39 & 3.2373 & 0.0001 & {$[0.997,3.26]$} \\
\hline \multirow{2}{*}{$<30$} & Male & 0 & 2 & - & - & \\
\hline & Female & 8 & 9 & 1.4889 & 0.559 & {$[-3.15,4.15]$} \\
\hline \multirow{2}{*}{ 30-40 } & Male & 2 & 3 & 6.7589 & 0.053 & . \\
\hline & Female & 7 & 15 & 2.1774 & 0.118 & {$[-1.27,3.90]$} \\
\hline \multirow{2}{*}{$>40$} & Male & 11 & 6 & 2.8995 & 0.162 & {$[-1.11,5.44]$} \\
\hline & Female & 22 & 15 & 5.0111 & 0.0004 & {$[1.46,4.54]$} \\
\hline
\end{tabular}

Table 4. sRAGE plasma concentration in total MS patients and age-/sex-related subgroups compared with corresponding healthy subjects. a: Base on Bootstrap sampling method

\begin{tabular}{|c|c|c|c|c|c|c|c|}
\hline & & Controls & MS patient & Mean Difference & Standard Error & Pvalue & $95 \%$ confidence Interval \\
\hline Total & & 50 & 50 & 1.725 & 0.348 & $<0.0001$ & {$[1.03,2.41]$} \\
\hline Male & & 11 & 6 & 2.15 & 0.39 & $<0.0001$ & {$[1.33,2.98]$} \\
\hline Femal & & 39 & 44 & 1.57 & 0.34 & $<0.0001$ & {$[0.88,2.27]$} \\
\hline \multirow{2}{*}{$<30$} & Male & 4 & 1 & - & - & - & - \\
\hline & Female & 15 & 10 & 1.12 & 0.8 & 0.184 & {$[0.59,2.8]^{2}$} \\
\hline \multirow{2}{*}{$30-40$} & Male & 3 & 4 & 2 & 0.53 & 0.034 & {$[0.28,3.71]^{2}$} \\
\hline & Female & 6 & 14 & 0.72 & 0.62 & 0.25 & {$[0.56,2.02]^{2}$} \\
\hline \multirow{2}{*}{$>40$} & Male & 4 & 1 & 2.32 & 0.55 & 0.01 & {$[1.14,3.5]^{2}$} \\
\hline & Female & 18 & 20 & 2.2 & 0.49 & $<0.0001$ & {$[1.19,3.21]^{\mathrm{a}}$} \\
\hline
\end{tabular}

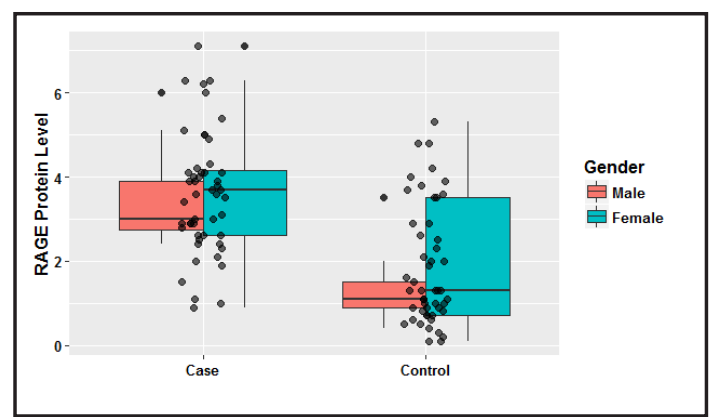

Fig. 2. sRAGE concentrations in MS patients treated with IFN1 $\beta$ and healthy subjects.

Table 5. Multiple linear regression model used for analysis of the correlations between independent variables and sRAGE levels. CI: confidence interval

\begin{tabular}{ccccccl}
\hline & Standardized Beta Coefficient & T & Partial Correlation & $95 \%$ CI for Beta & P value \\
\hline Age & 0.271 & 0.374 & 0.056 & -0.173 & 0.252 & 0.71 \\
EDSS & -0.282 & -2.149 & -0.308 & -0.717 & -0.023 & 0.037 \\
Disease duration & -0.006 & -0.015 & -0.002 & -0.211 & 0.208 & 0.988 \\
Age at onset & -0.29 & -0.383 & -0.058 & -0.25 & 0.17 & 0.703 \\
& & & & & & 0.535 \\
sex & -0.104 & -0.626 & -0.094 & -1.481 & 0.78 &
\end{tabular}


Fig. 3. Correlation between RAGE transcript levels and sRAGE plasma concentrations (a: Spearman correlation coefficient).

treated with CinnoVex than in healthy subjects. A recent study has shown up-regulation of RAGE transcript levels in MS patients following treatment with IFN $\beta$-1a and

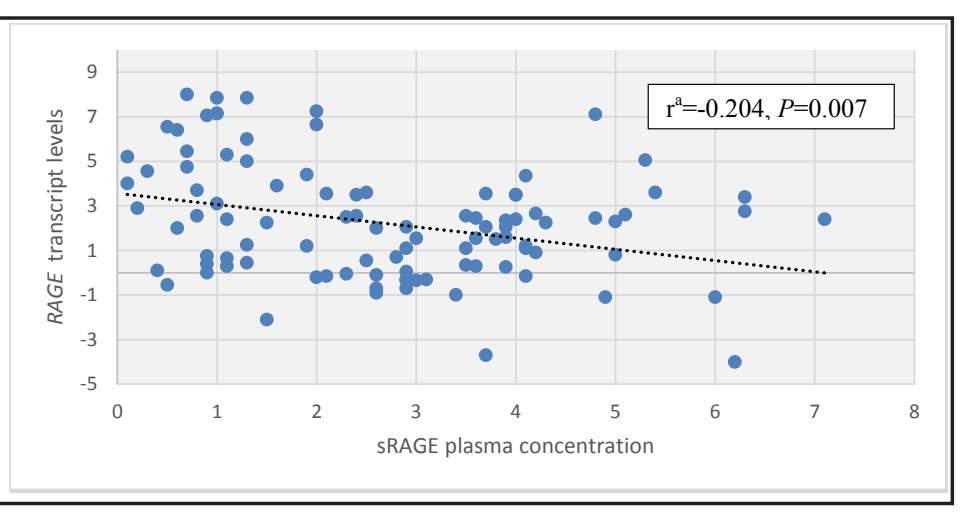
suggested RAGE up-regulation as a mechanism by which IFN $\beta$-1a alters immune responses [14]. In the current study, considering the location of primers as well as the fact that the extracellular domain is shared between RAGE and sRAGE [12] and these two proteins exert opposing effects on immune response, the functional importance of over-expression of the related transcript cannot be inferred from transcript studies.

A commercial ELISA kit was used to demonstrate the significant higher levels of sRAGE in MS patients treated with CinnoVex compared with healthy subjects. The role of sRAGE in decreasing the inflammatory response and improving numerous inflammatory diseases has been described previously $[15,16]$. A decrease in sRAGE has also been associated with disease severity in axonal Guillain-Barré syndrome [12]. As in our cohort of patients, all MS patients were responsive to CinnoVex, we propose up-regulation of sRAGE as a possible mechanism for clinical response to this kind of treatment. Although the exact mechanism of the effect of IFN $\beta-1$ a on sRAGE was not assessed in the present study, we suggest that such an effect might be exerted through modulation of RAGE mRNA alternative splicing or by proteolytic cleavage of the membrane-bound RAGE protein leading to changes in the RAGE/sRAGE ratio. IFN $\beta$ treatment has been previously shown to influence expression of certain TRAIL- $\alpha$ and TRAILR-2 isoforms [17]. However, other possibilities such as ligandlike behavior of IFN $\beta$-1a or the presence of other ligands in the blood cannot be excluded.

Sternberg et al. compared serum levels of sRAGE between 37 MS patients and 22 healthy control subjects and found significantly higher down-regulation of sRAGE in MS patients. They also detected a relationship between sRAGE and EDSS as well as SRAGE and the rate of clinical relapse in MS patients [18]. The population of patients recruited in the current study was remarkably different from those in the Sternberg et al. study, who included 31 RRMS and six secondary progressive (SP) MS patients. This differs from our homogenized patient population consisting of 50 RRMS patients. More importantly, Sternberg et al. enrolled patients who declined to take any Food and Drug Administration approved MS diseasemodifying drugs (IFN- $\beta$, glatiramer acetate and natalizumab) [18], which could explain the discrepancies between our results and theirs.

Glasnović et al. compared sRAGE level and RAGE transcript levels in the peripheral blood and cerebrospinal fluid (CSF) of 27 newly diagnosed MS patients with those of healthy subjects and found lower levels of sRAGE in the CSF of MS patients [19]. Dorota et al. evaluated serum levels of sRAGE in 44 MS patients (including both rapidly and slowly progressive patients) and found significantly increased levels of sRAGE in the serum of MS patients when compared to controls [20]. Another study found a higher percentage of membrane-bound RAGE-positive on the peripheral blood mononuclear cells (PBMCs), monocytes and T cells of MS patients when compared with healthy subjects. In addition, they detected lower percentages of RAGE-positive monocytes and RAGE-positive T cells in SPMS patients compared with RRMS patients. Decreased membrane bound RAGE expression on PBMCs, especially on monocytes, was suggested by the authors as a biomarker of MS disease severity and progression [21]. 
Taken together, the data presented above illustrates the anti-inflammatory effect of sRAGE, but the pro-inflammatory effects of membrane-bound RAGE in MS and supports the association between IFN $\beta-1$ a treatment and increased sRAGE concentration. Such a proposition is supported by the results of animal studies which have demonstrated suppression of EAE through inhibition of RAGE [11]. In addition, the inverse correlation between SRAGE plasma concentrations and EDSS as revealed in the current study is in line with such hypothesis.

Considering the stable clinical state of MS patients in the current study and their response to CinnoVex, the elevated levels of sRAGE in patients compared with healthy subjects might be due to the effects of this kind of treatment. However, the existence of variable sources for sRAGE production in the body indicate that future studies should be performed to assess this hypothesis.

The current study has some limitations, including lack of analysis of sRAGE levels in CSF samples due to unavailability of CSF samples from healthy subjects. Moreover, in the present study, we used a commercial ELISA kit that measures the total pool of sRAGE by means of antibodies that spot both the spliced and cleaved forms of sRAGE. Although it is possible to measure splice-variant endogenous secretory RAGE (esRAGE) in serum because of its distinctive C-terminus sequence [22], the results of the previous studies indicate an association between total sRAGE levels and inflammatory processes [23] as well as a significant correlation between sRAGE and esRAGE levels [24]. The current study, thus, aimed to assess total sRAGE levels. Future studies should measure the esRAGE levels to evaluate this possibility.

\section{Acknowledgements}

The current study was supported by a grant from Shahid Beheshti University of Medical Sciences.

\section{Disclosure Statement}

No conflict of interests exists.

\section{References}

1 Eftekharian MM, Ghafouri-Fard S, Soudyab M, Omrani MD, Rahimi M, Sayad A, Komaki A, Mazdeh M, Taheri M: Expression Analysis of Long Non-coding RNAs in the Blood of Multiple Sclerosis Patients. J Mol Neurosci 2017;10.1007/s12031-017-0982-1

-2 Eftekharian MM, Azimi T, Ghafouri-Fard S, Sayad A, Omrani MD, Sarrafzadeh S, Abbasalipourkabir R, Mazdeh M, Taheri M: Phospholipase D1 expression analysis in relapsing-remitting multiple sclerosis patients. Neurol Sci 2017;38:865-872.

-3 Sayad A, Ghafouri-Fard S, Omrani MD, Noroozi R, Taheri M: Myxovirus resistance protein A (MxA) polymorphism is associated with IFNbeta response in Iranian multiple sclerosis patients. Neurol Sci 2017;38:1093-1099.

4 Taheri M, Ghafouri-Fard S, Solgi G, Sayad A, Mazdeh M, Omrani MD: Determination of cytokine levels in multiple sclerosis patients and their relevance with patients' response to Cinnovex. Cytokine 2017;96:138143.

5 Chuah YK, Basir R, Talib H, Tie TH, Nordin N: Receptor for advanced glycation end products and its involvement in inflammatory diseases. Int J Inflam 2013;2013:403460.

6 Brett J, Schmidt AM, Yan SD, Zou YS, Weidman E, Pinsky D, Nowygrod R, Neeper M, Przysiecki C, Shaw A, et al.: Survey of the distribution of a newly characterized receptor for advanced glycation end products in tissues. Am J Pathol 1993;143:1699-1712. 


\section{Cellular Physiology Cell Physiol Biochem 2018;46:561-567

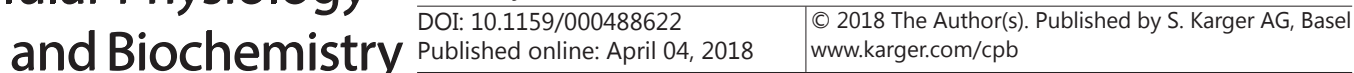

7 Mene P, Festuccia F, Pugliese F: Clinical potential of advanced glycation end-product inhibitors in diabetes mellitus. Am J Cardiovasc Drugs 2003;3:315-320.

-8 Lanati N, Emanuele E, Brondino N, Geroldi D: Soluble RAGE-modulating drugs: state-of-the-art and future perspectives for targeting vascular inflammation. Curr Vasc Pharmacol 2010;8:86-92.

-9 Bodine BG, Bennion BG, Leatham E, Jimenez FR, Wright AJ, Jergensen ZR, Erickson CJ, Jones CM, Johnson JP, Knapp SM, Reynolds PR: Conditionally induced RAGE expression by proximal airway epithelial cells in transgenic mice causes lung inflammation. Respir Res 2014;15:133.

10 Saha A, Kim S-J, Zhang Z, Lee Y-C, Tsai P-C, Mukherjee AB: Elevated expression of S100B and RAGE positively correlates with pro-inflammatory cytokine production that may contributie to rapid neurodegeneration in INCL. FASEB J 2007;21:A989.

11 Yan SS, Wu ZY, Zhang HP, Furtado G, Chen X, Yan SF, Schmidt AM, Brown C, Stern A, LaFaille J, Chess L, Stern DM, Jiang H: Suppression of experimental autoimmune encephalomyelitis by selective blockade of encephalitogenic T-cell infiltration of the central nervous system. Nat Med 2003;9:287-293.

12 Zhang D-Q Wang R, Li T, Zhou J-P, Chang G-Q Zhao N, Yang L-N, Zhai H, Yang L: Reduced soluble RAGE is associated with disease severity of axonal Guillain-Barré syndrome. Sci Rep 2016;6:21890.

13 Polman CH, Reingold SC, Banwell B, Clanet M, Cohen JA, Filippi M, Fujihara K, Havrdova E, Hutchinson M, Kappos L, Lublin FD, Montalban X, O’Connor P, Sandberg-Wollheim M, Thompson AJ, Waubant E, Weinshenker B, Wolinsky JS: Diagnostic Criteria for Multiple Sclerosis: 2010 Revisions to the McDonald Criteria. Ann Neurol 2011;69:292-302.

14 Asadikaram G, Noroozi S, Ebrahimi Meimand HA, Sanjari M, Zainodini N, Khoramdelazad H, Shahrokhi N, Kazemi Arababadi M: Interferon-beta 1a Modulates Expression of RAGE but Not S100A12 and Nuclear Factor-kappaB in Multiple Sclerosis Patients. Neuroimmunomodulation 2016;23:345-351.

15 Koyama H, Yamamoto H, Nishizawa Y: RAGE and soluble RAGE: potential therapeutic targets for cardiovascular diseases. Mol Med 2007;13:625-635.

16 Yan SF, Ramasamy R, Schmidt AM: Soluble RAGE: therapy and biomarker in unraveling the RAGE axis in chronic disease and aging. Biochem Pharmacol 2010;79:1379-1386.

17 López-Gómez C, Oliver-Martos B, Pinto-Medel M-J, Suardiaz M, Reyes-Garrido V, Urbaneja P, Fernández Ó, Leyva L: TRAIL and TRAIL receptors splice variants during long-term interferon $\beta$ treatment of patients with multiple sclerosis: evaluation as biomarkers for therapeutic response. J Neurol Neurosurg Psychiatry 2015;jnnp-2014-309932.

18 Sternberg Z, Weinstock-Guttman B, Hojnacki D, Zamboni P, Zivadinov R, Chadha K, Lieberman A, Kazim L, Drake A, Rocco P, Grazioli E, Munschauer F: Soluble receptor for advanced glycation end products in multiple sclerosis: a potential marker of disease severity. Mult Scler 2008;14:759-763.

19 Glasnovic A, Cvija H, Stojic M, Tudoric-Deno I, Ivcevic S, Romic D, Ticinovic N, Vuletic V, Lazibat I, Grcevic D: Decreased level of sRAGE in the cerebrospinal fluid of multiple sclerosis patients at clinical onset. Neuroimmunomodulation 2014;21:226-233.

20 Dobrota D, Cierny D, Michalik J, Kurca E, Lehotsky J: sRage plasma level in association with multiple sclerosis risk in Slovak population. SpringerPlus 2015;4:P10.

-21 Sternberg Z, Chiotti A, Tario J, Chichelli T, Patel N, Chadha K, Yu J, Karmon Y: Reduced expression of membrane-bound (m)RAGE is a biomarker of multiple sclerosis disease progression. Immunobiology 2016;221:193-198.

-22 Kalea AZ, Schmidt AM, Hudson BI: Alternative splicing of RAGE: roles in biology and disease. Front Biosci 2011;16:2756-2770.

23 Gopal P, Reynaert NL, Scheijen JL, Schalkwijk CG, Franssen FM, Wouters EF, Rutten EP: Association of plasma sRAGE, but not esRAGE with lung function impairment in COPD. Respir Res 2014;15:24.

24 Yang SJ, Kim S, Hwang SY, Kim TN, Choi HY, Yoo HJ, Seo JA, Kim SG, Kim NH, Baik SH, Choi DS, Choi KM: Association between sRAGE, esRAGE levels and vascular inflammation: analysis with (18) F-fluorodeoxyglucose positron emission tomography. Atherosclerosis 2012;220:402-406. 